\title{
Olive separation machine based on image processing
}

\author{
M. Kuncan*, H.M. Ertunç** \\ *Kocaeli University, Umuttepe Campus, Engineering Faculty, Department of Mechatronic Engineering, 41380, Izmit, \\ Kocaeli, Turkey E-mail: melih.kuncan@kocaeli.edu.tr \\ **Kocaeli University, Umuttepe Campus, Engineering Faculty, Department of Mechatronic Engineering, 41380, Izmit, \\ Kocaeli, TurkeyE-mail: hmertunc@kocaeli.edu.tr
}

cross $^{\text {ref }}$ http://dx.doi.org/10.5755/j01.mech.22.5.13726

\section{Introduction}

The necessity of new applications in industrial agriculture has become crucial as the technology develop in the last years. The prolificacy in some industrial agriculture applications can be increased by using image processing techniques. Many devices based on modern technology were designed in industrial agriculture that is more effective than labor force. Automation systems have many advantages such as high precision, high process capability, non-contact detection etc. Therefore, image processing based systems have become popular in industrial agriculture in recent years [1-3].

In the literature, various agriculture systems based on image processing techniques have been developed. Some researchers developed a watermelon ripeness detection system based on image processing and Artificial Neural Networks (ANN). They used YCbCr Color space instead of Red, Green, and Blue (RGB) color space. After the pre-processing of the images, they determined the sum of each color chrominance $(\mathrm{Cb}$ and $\mathrm{Cr})$ as a feature. They detected ripeness of watermelon with \% 86 accuracy [4]. In another research, image processing based pear external quality system was developed for detecting shape, color and surface defect. They used Hue, Saturation, Intensity (HIS) color space instead of RGB color space for detecting color. They used histogram of Hue value of each images for detecting the color [5]. Xu Yuanfang and his team developed an image processing based system which monitors maize leaf nutrition. As a pre-processing operation they used a median filter $(3 \times 3$ templates) for smoothing the images. After median filtering, they used white balance algorithm for restoring image's white background into pure white. For color detection, they used the mean and standard deviation of each color channel $(R, G, B)$ for a feature set. After the feature extraction, they used regression analysis for detection of maize leaf nutrition [6].

Olive production is farmed mostly in Aegean region of Turkey due to its geological position. After the harvest, olives are separated by labors according to their color as shown in Fig. 1. Nearly one ton of olives could be separated by five labors in a day. The system developed in this work can separate about 30 tons of olives in a day. On the other hand, hygiene of human health is very important in food sector. The developed system offers a more hygienic and fast solution for olive separation process than un-reliable manual separation of olives. Developed system gets more accurate results than a human based system.

In this study, a process for olive separation based on the color was examined. A set-up was designed for that purpose and the developed image processing algorithm was tested with the set-up in real time. The objective of this research is to apply different image processing techniques to separate the olives according to their colors. The study tested for three different methods in image processing part and the success of these methods were compared in the results section. It was observed that the algorithm performed successfully and detected the olives. The paper first discusses the automation system in agriculture. The second section deals with image processing algorithm. The mechanical and electronic designs were explained in the third section. Then, experimental results are presented in Section 4.

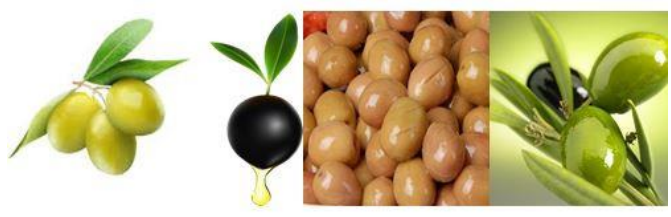

Fig. 1 Different olives according to harvest

\section{Image processing algorithm}

In this study, an image processing-based system for olive separation has been developed. For carrying out image processing, a Ueye-KP20B type camera has been integrated into the system. The camera images were processed by the image processing algorithm that determines the color of olives. After image processing, output data is obtained. The output data from the system is transferred to valve control card in real time via a computer serial port. The properties of the camera used in olive separation process are given in Table 1.

Table 1

The Properties of Camera

\begin{tabular}{|l|l|}
\hline Optical class & 1 \\
\hline Interface & USB 3.0 \\
\hline Sensor Type & CMOS \\
\hline Frame Rate & $60 \mathrm{fps}$ \\
\hline Resolution & $1280 \times 1024$ \\
\hline
\end{tabular}

\subsection{The image processing algorithms}

The image processing algorithms developed in this section are explained. For image processing applications, HSV (Hue, Saturation, Value) for distinguishing colored objects by using color model method and RGB (Red, Green, 
Blue) color model to distinguish colored objects were performed based on Euclidean and Mahalanobis distance method. In part of RGB color model, the results obtained from background and feature extraction methods were also compared for this application.

\subsubsection{HSV and RGB color spaces}

HSV color space defines colors as hue, saturation and brightness, respectively. This method is preferable because HSV color space has a structure that is closer to the human visual mechanism as compared to the RGB space. Generally, HSV color space model has been used for distinguishing colored objects. Although all components affect brightness in RGB space, only V component value affects brightness in HSV space [7].

A median filter was applied to the images as a preprocessing operation for smoothing the image and removing the noise on image. After the filtering operation, the format of the image was changed from RGB to HSV. HSV color space is used frequently in color detection algorithm because of its cylindrical form. RGB and HSV color spaces were given in Fig. 2.
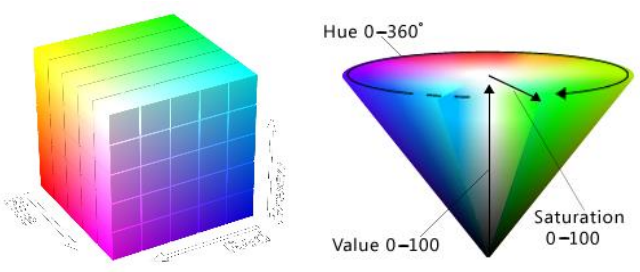

Fig. 2 RGB and HSV color spaces

After this color space conversion, a pre-determined threshold was applied to the image. Before sample image and after Saturation process image was shown in Fig. 3.

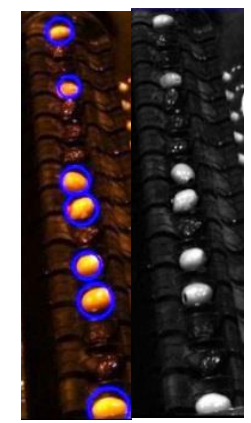

Fig. 3 Frames before and after Saturation process

After the Saturation process operation, a threshold value was applied to the image. This threshold was determined by using Saturation histogram of the image. A sample image and its Saturation histogram were shown in Fig. 4.

A threshold determined by trial and error was applied to images transformed from RGB to HSV space. Olives whose Hue and Saturation values above the determined threshold were accepted as green olives.

A labeling algorithm was applied to the image and each green olive was detected in a tumbler. Then mean value of Saturation channel at detected olive was selected as a feature. The Euclidean distance between detected olive image and sample olive image was calculated. If Euclidean distance of detected olive is greater than a pre-determined threshold, the olive can be assumed as green.

The olives were placed equally spaced on each tumbler. Olive position which has minimum row index in the tumbler was assumed as starting position. Other olives positions were detected depending on the row index. Valves mounted on mechanical system at related position were opened by electronic card according to received data. Sample image and detected olive positions were shown in Fig. 5.

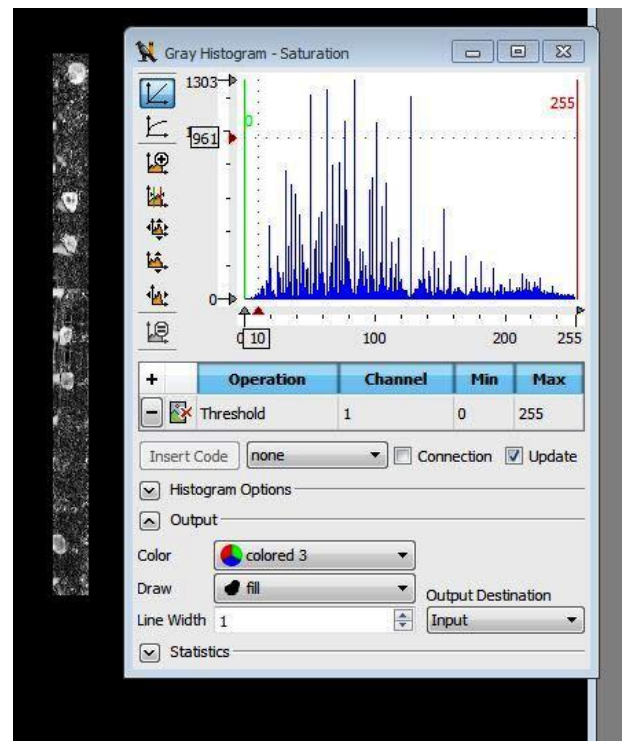

Fig. 4 A sample image and its Saturation histogram

\subsubsection{A classification method based on Euclidean distance in the RGB color space}

Classification is a decision-making process that is used in many disciplines. The classification process is based on similarity of object in a particular class and it can be defined as grouping according their properties in a data set. On the other hand, images features should be extracted to perform a good classification using image processing techniques. In this study, the averages of the images in RGB space at each R, G, B channels were calculated and considered as features. Therefore, a three-dimensional feature space was obtained. On the other hand, various difference measurement techniques for classification process are employed in this study. Among them, Euclidean distance was chosen that is the most commonly preferred method due to its easy application.

The classification process was performed according to olive color. These classification colors are light green, green, dark green and black. The classes were created by obtaining 40 different samples from each class. The average of each channel in RGB image space was selected as a feature. Therefore, three-dimensional space consisting of $40 \mathrm{el}-$ ements was obtained.

Euclidean distance was calculated according to the Eq. 1. Olives in received image are included to closest olive cluster according to obtained values using the following formula [8]:

$$
\sqrt{\left(p_{1}-q_{1}\right)^{2}+\ldots+\left(p_{n}-q_{n}\right)^{2}}=\sqrt{\sum_{i=1}^{n}\left(p_{i}-q_{i}\right)^{2}},
$$

where $p_{i}$ and $q_{i}$ are pixel points, respectively. This distance method does not take into account information except the 
two points. Euclidean distance formula given in Eq. 1 is used for this classification method to detect closest distance in two points. Euclidean distance calculates linear distance of one point to the other point by obtaining values with Pythagorean Theorem. Algorithm compares new data by scanning the entire data set and olive was assigned to the closest cluster. The sample image for Euclidian distance method in RGB color space is shown in Fig. 5.
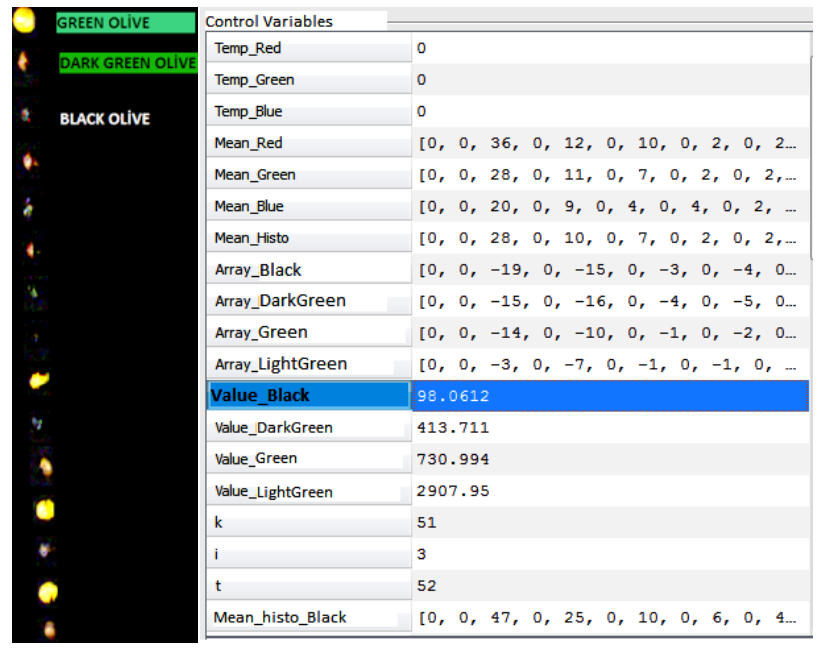

Fig. 5 Sample image in RGB color space for Euclidean method

\subsubsection{Mahalanobis distance method in the RGB color space with classification}

Euclidean distance is the most basic method to measure the distance between two points in space. Euclidean distance take into account information only distance between two points. However, the distance to the center may not be adequate in some cases. Mahalanobis distance method considers the distance to the center with standard deviation values. Thus, the classification process is performed with a higher accuracy.

In order to perform Mahalanobis method in image processing applications, specific clusters are required to determine as done in the Euclidean distance method. For each cluster which is light green, green, dark green and black olives, 40 different samples are used for determining the cluster. For a sample olive clusters, the average of the RGB value is used. Standard deviations are calculated in the same way as examples algorithm. The first step in this method is to obtain the sample sets for comparison. This step can be called as the stage of the learning of the object. Firstly, clusters to be sampled are determined. The pixel mean and standard deviation values in RGB space is used in Mahalanobis distance formula. Olive clusters can be determined with this formula. When the Mahalanobis distance formula applied for comparing olive clusters, a Mahalanobis distance for each sample cluster is obtained. As a result of this process, olives are assigned to clusters according to the lowest Mahalanobis distance. The sample image for Mahalanobis distance method in RGB color space is shown in Fig. 6.

The general Mahalanobis formula is shown in Eq. 2 where $x_{i}$ is average $\mathrm{R}, \mathrm{G}$ and $\mathrm{B}$ value of the processed image and $m_{i}$ is average $\mathrm{R}, \mathrm{G}$ and $\mathrm{B}$ value of sample olive image used in classification. Eq. 3 shows abbreviated state of the previous equation. The Eq. 4 shows open state form of Eq. 3 [9].
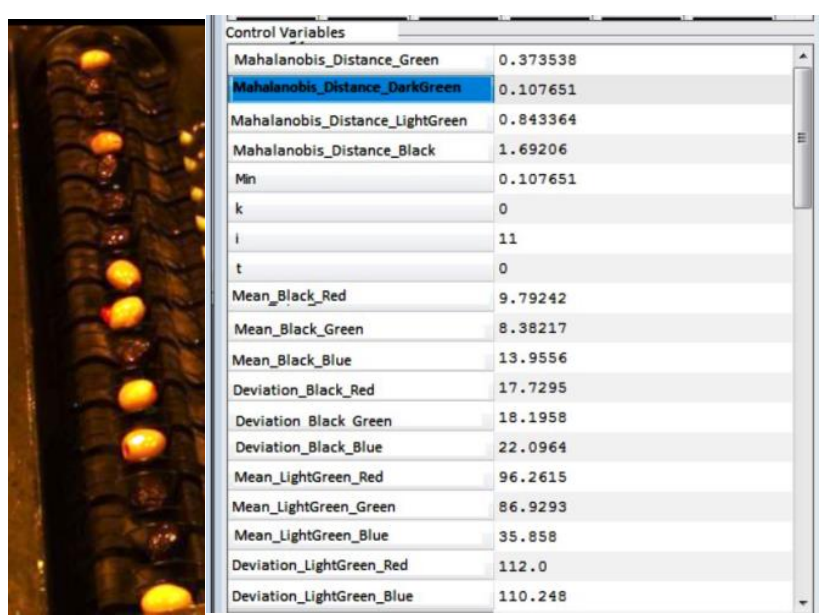

Fig. 6 Sample image in RGB color space for the Mahalanobis method

$$
\begin{aligned}
& D(x, y)=\sqrt{\left(\left[\begin{array}{c}
x_{1} \\
x_{2} \\
\vdots \\
x_{n}
\end{array}\right]-\left[\begin{array}{c}
m_{1} \\
m_{2} \\
\vdots \\
m_{3}
\end{array}\right]\right) S^{-1}\left(\left[\begin{array}{c}
x_{1} \\
\vdots \\
x_{n}
\end{array}\right]^{T}-\left[\begin{array}{c}
m_{1} \\
\vdots \\
m_{n}
\end{array}\right]^{T}\right)} \\
& D_{M}(x)=\sqrt{(x-m)^{T} S^{-1}(x-m)} \\
& {[x-m]=\left[\left(x_{R}-m_{R}\right)\left(x_{G}-m_{G}\right)\left(x_{B}-m_{B}\right)\right] .}
\end{aligned}
$$

Eq. 4 is the first component of the Mahalanobis formula. The covariance matrix of Mahalanobis formula requires to be transformed according to olive separation algorithm. The covariance matrix is given in Eq. 5 .

$$
S=\left[\begin{array}{lll}
\sigma_{R}^{2} & 0 & 0 \\
0 & \sigma_{G}^{2} & 0 \\
0 & 0 & \sigma_{B}^{2}
\end{array}\right] .
$$

As in the custom Mahalanobis formula, inverse of the $\mathrm{S}$-covariance matrix is calculated as given in Eq. 6 .

$$
S^{-1}=\left[\begin{array}{cccc}
1 / \sigma_{R}^{2} & 0 & 0 \\
0 & 1 / \sigma_{G}^{2} & 0 \\
0 & 0 & 1 / \sigma_{B}^{2}
\end{array}\right] .
$$

The last argument of the Mahalanobis distance method is calculated as shown in Eq. 7.

$$
[x-m]^{T}=\left[\begin{array}{c}
x_{R}-m_{R} \\
x_{G}-m_{G} \\
x_{B}-m_{B}
\end{array}\right] .
$$

The parameters found are used in Eq. 8: 


$$
D(x, m)=\sqrt{[x-m]\left[\begin{array}{cccc}
1 / \sigma_{R}^{2} & 0 & 0 \\
0 & 1 / \sigma_{G}^{2} & 0 \\
0 & 0 & 1 / & \sigma_{B}^{2}
\end{array}\right][x-m]^{T}} .
$$

After implementing the necessary operations in the Eq. 8, Mahalanobis formula used in this study is finally transformed into the following Eq. (9).

$$
D=\left[\frac{\left(x_{R}-m_{R}\right)^{2}}{\sigma_{R}^{2}}+\frac{\left(x_{G}-m_{G}\right)^{2}}{\sigma_{G}^{2}}+\frac{\left(x_{B}-m_{B}\right)^{2}}{\sigma_{B}^{2}}\right]^{\frac{1}{2}} \text {. }
$$

\section{Mechanical and electronical design}

In this study, a platform for an olive separation system was designed and realized. Mechanical design of the platform was implemented at SolidWorks environment with considering the system constraints. The mechanical part consists of motors, valves, and five independent tumblers. 15 olives were placed at each tumbler. 75 olives could be placed in the system at same time. The technical drawing of the mechanical system is illustrated in Fig. 7 and the picture of the tumblers of the mechanical part of the system was given in Fig. 8.

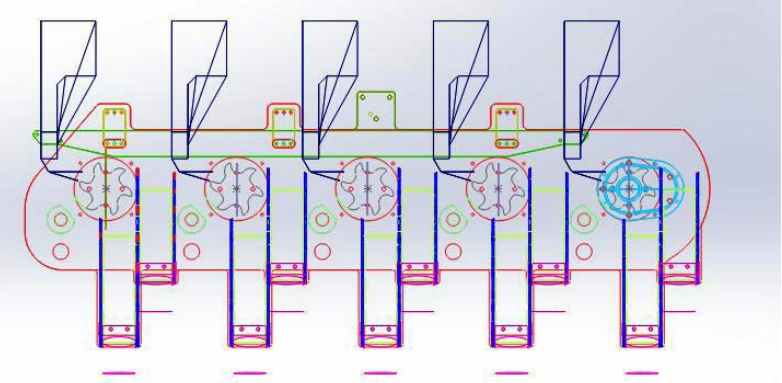

a

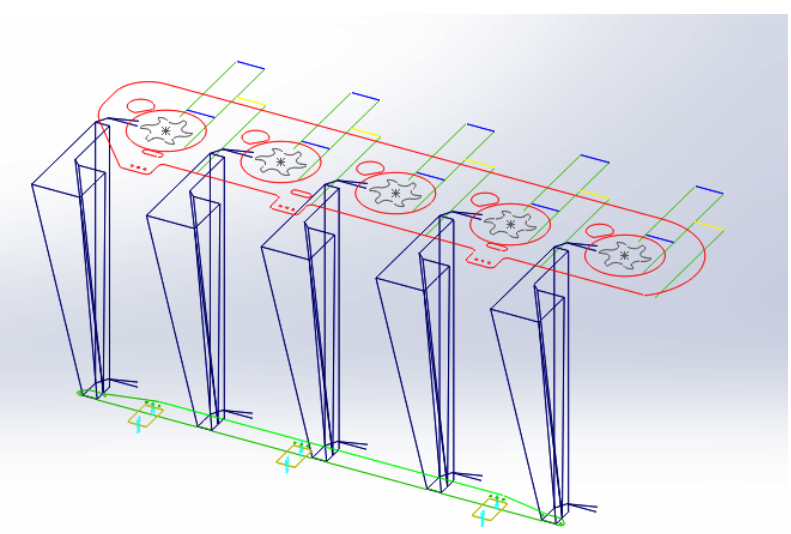

b

Fig. 7 Technical drawing of the system: a-front view; b- isometric view

An electronic controller card of the system was designed at Altium environment. The electronic part consists of a microcontroller and Darlington transistors. All supply pins of each valve connected to the same line and ground pin of each valve connected to transistor outputs. When data arrived to controller via serial port, controller opens or closes related valve according to the command. Schematic design and its printed form of designed circuit were given in Fig. 9.

The color of the olives is determined based on the image processing algorithm developed in this study. The air valves are used to throw olives according to their colors. These valves are mounted to the mechanical blower for providing air flow. These valves transfer olives to the desired appropriate area. The valve control card was designed for communicating with image proccesing algorithm. This valve control card is supported by RS-232 communication.

The data is transmitted to the valves in the specified location by the serial port and related valve is activated. Thus, olive separation process is carried out with air. 3/2 solenoid valves are used in the system. The valves used in the separation system are closed normally. They are opened by triggering with 0.5 bar pressure and keep working up to 10 bar pressure. In the experiments of the system, ideal pressure 2.2 bar was determined by trial and error.
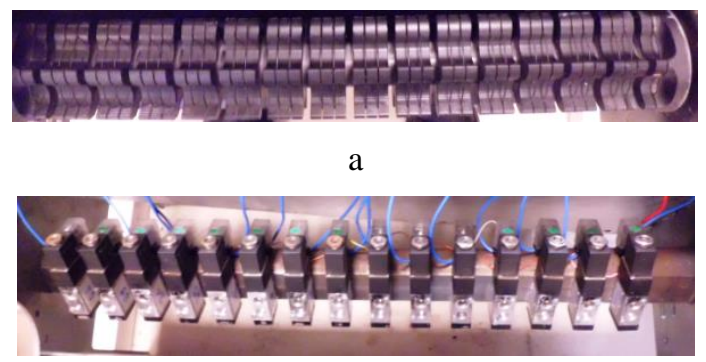

$\mathrm{b}$

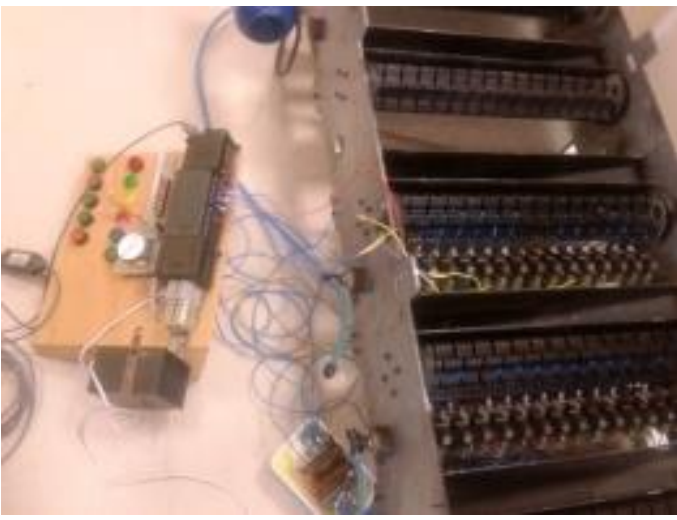

Fig. 8 The tumblers of the mechanical part of the system: $\mathrm{a}$-tumbler; b-selenoid valves; $\mathrm{c}$ - the system assembly

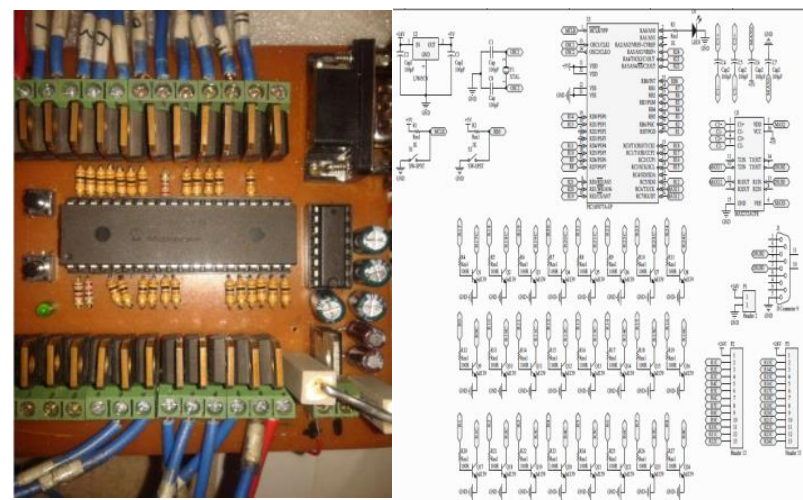

Fig. 9 Electronic design of system and it's printed form 


\section{Experimental results}

In this study, an image processing based olive color separation machine was developed. Green olives were separated from other colored olives that are not green. Different image processing methods were used to separate the olives and their advantages and/or disadvantages are discussed. Three different methods are used in the classification process according to the color of the object in the software part. These methods are HSV color space, Euclidian distance with RGB color space and Mahalonobis distance with RGB color space. Background subtraction and feature extraction methods are used in the Euclidian distance and Mahalonobis distance. Classification was applied based on feature values. Four different classes are defined according to the color of olives. These classes are light green, green, dark green and black. Many different samples were used for each class. The success rate for different classes is shown in Fig. 10.

A success rate with $90 \%$ was achieved for HSV space method, while $80 \%$ success rate for Euclidean distance method and $97 \%$ success rate for Mahalanobis distance method were obtained. Each method is realized for four color clasess method. The percentages of olive color class success chart for each method are shown in Fig. 11.

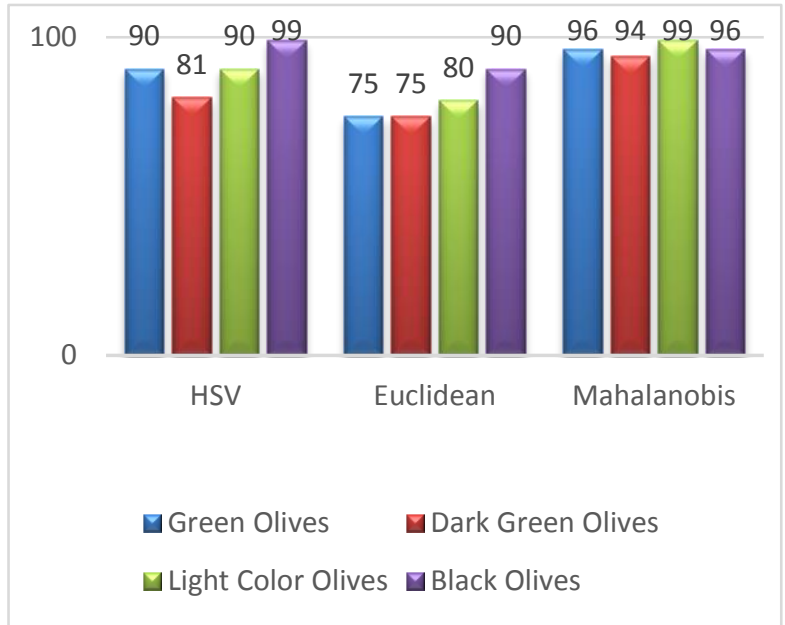

Fig. 10 The developed image processing methods for the classification process the success rates

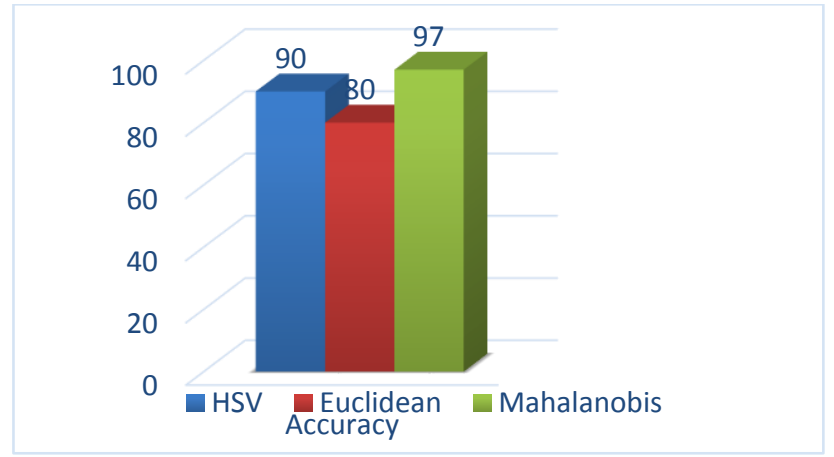

Fig. 11 The success rate of three methods

Run-time is also very important especially for the real time industrial applications. Therefore, run-time of three methods was compared in Table 2.

Mahalanobis distance is observed as the most successful method out of three different image processing methods. The method based on Mahalanobis distance has more accuracy because of using standard deviation parameter. On the other hand, HSV space seems to be successful more than Euclidean distance method. Although the low success rate of Euclidean distance, it can be used in the classification process in practice because of its short run-time.

Table 2

Comparison of three different methods run-time

\begin{tabular}{|l|l|l|l|}
\hline & \multicolumn{1}{|c|}{ HSV } & \multicolumn{1}{|c|}{ Euclidean } & \multicolumn{1}{|c|}{ Mahalanobis } \\
\hline Time, sec & 0,7986 & 1,0406 & 0,9234 \\
\hline
\end{tabular}

\section{Conclusions}

Many different type of automation systems were developed in industrial agriculture based on image processing techniques. On the other hand, it is very important to realize agricultural industrial system in real time. An olive separation machine was designed and developed in this study that can be potentially used as an industrial product. Firstly, mechanical plots of a prototype are designed at computer environment and a setup was manufactured for this research. Different real color olive images were collected by camera. Image processing methods are developed to classify olives. In order to increase success rate of image processing algorithm, HSV, Euclidian and Mahalanobis distance methods were used. Among them, Mahalanobis distance method outperformed the others with $97 \%$ success rate.

In this study, image processing algorithms were applied to perform olive classification based on their colors. Therefore, a separation machine has been designed and manufactured. The studies and developed models can be adapted to other agricultural industrial applications.

\section{Acknowledgment}

This work has been realized at Sensor Laboratory in Kocaeli University and was supported by Kocaeli University Scientific Research Projects Coordination Unit Coordinator BAP under the project code 013-014. The authors thanks to Sensor Laboratory Research and Development group for their contributions.

\section{References}

1. Guijarro, M.; Riomoros, I.; Pajares, G.; Zitinski, P. 2015. Discrete wavelets transform for improving greenness image segmentation in agricultural images, Computers and Electronics in Agriculture 118: 396-407. http://dx.doi.org/10.1016/j.compag.2015.09.011.

2. Wenmei, L.; Yuzhen, L. 2010. Aquaculture monitoring system, In Information Technology and Applications (IFITA), 2010 International Forum on 2: 138-141. http://dx.doi.org/10.1109/IFITA.2010.291.

3. Hufschmied, P.; Fankhauser, T.; Pugovkin, D. 2011. Automatic stress - free sorting of sturgeons inside culture tanks using image processing, Journal of Applied Ichthyology 27(2): 622-626. http://dx.doi.org/10.1111/j.1439-0426.2011.01704.x.

4. Shah Rizam, M.S.B.; Farah Yasmin, A.R.; Ahmad Ihsan, M.Y.; Shazana, K. 2009. Non-destructive watermelon ripeness determination using image processing 
and artificial neural network (ANN), International Journal of Electrical and Computer Engineering 3(2): 332336.

5. Zhao, Y.; Wang, D.; Qian, D. 2009. Machine vision based image analysis for the estimation of Pear external quality, In Intelligent Computation Technology and Automation, ICICTA'09, Second International Conference on 1: 629-632. http://dx.doi.org/10.1109/ICICTA.2009.157.

6. Yuanfang, X.; Xianmin, W.; Hong, S.; Haihua, W.; Yan'e, Z. 2010. Study of monitoring maize leaf nutrition based on image processing and spectral analysis, In World Automation Congress (WAC), 465-468.

7. Tonguç G. 2004. Görüntü İșleme Teknikleri Kullanılarak Meyve Tasnifi, Master Thesis, Süleyman Demirel Üniversitesi Fen Bilimleri Enstitüsü, Isparta, 200542.

8. Liwei, W.; Yan, Z.; Jufu, F. 2005. On the Euclidean distance of images, IEEE Transactions on Pattern Analysis and Machine Intelligence 27(8): 1334-1339. http://dx.doi.org/10.1109/TPAMI.2005.165.

9. Zhao, X.; Li, Y.; Zhao, Q. 2015. Mahalanobis distance based on fuzzy clustering algorithm for image segmentation, Digital Signal Processing 43(2015): 8-16. http://dx.doi.org/10.1016/j.dsp.2015.04.009.
M. Kuncan, H.M. Ertunç

\section{OLIVE SEPARATION MACHINE BASED ON IMAGE PROCESSING}

$\mathrm{S}$ u m m a r y

An olive seperation machine based on image processing has been developed in this study. For this purpose, mechanical and electronical design of the machine was performed. In the part of mechanical design, the air valves were used for separation of olives. The air valves were controlled with an electronic card according to output of the image processing algorithm. The position of the olives that were detected as black was switched and then the olives are thrown to a storage case. AN industrial digital camera was used for image processing, and an image processing algorithm is developed in Visual Basic 6.0 environment. The outputs of image processing algorithm were sent to electronic card by serial port. The image processing algorithm developed could process 30 frames per second. The image processing algorithm the system could separate 30 olives per second which is optimal and feasible for real time operation.

Keywords: real time process, olive separation machine, industrial agriculture, HSV and RGB color space, Mahalanobis and Euclidean distance, image processing algorithm.

Received November 30, 2015 Accepted September 28, 2016 